\title{
Beneficial effects of long-term liraglutide monotherapy on glycemic control and diabetic microangiopathies in a female Japanese diabetic patient with familial partial lipodystrophy 1
}

\author{
Iwanishi $\mathrm{M}^{1 *}$, Azuma $C^{1}$, Tezuka $\mathrm{Y}^{1}$, Yamamoto $\mathrm{Y}^{1}$, Ito-Kobayashi $\mathrm{J}^{1}$, Washiyama $\mathrm{M}^{1}$, Shimatsu $\mathrm{A}^{1}$, Mizuno $\mathrm{A}^{2}$ and Osafune $\mathrm{Y}^{2}$ \\ ${ }^{1}$ Department of Diabetes and Endocrinology, Kusatsu General Hospital 1660 Yabase, Kusatsu, Shiga 525-8585, Japan \\ ${ }^{2}$ Department of Ophthalmology, Kusatsu General Hospital 1660 Yabase, Kusatsu, Shiga 525-8585, Japan
}

\begin{abstract}
We experienced the case that a Japanese female diabetic patient with partial lipodystrophy continued liraglutide monotherapy for a long period on her will. At first examination, she had poor glycemic control and body weight reduction with decreased fat mass had been caused by uncontrolled diabetes. She had severe nonproliferative diabetic retinopathy and nephropathy with microalbuminuria. When she had weight gain after her glycemic control was improved, she still had fat loss in the lower limbs with abdominal fat excess. She had insulin deficiency and relatively severe insulin resistance. According to the clinical findings and genetic analysis, she might fit the category of familial partial lipodystrophy (FPLD)1. We administered liraglutide for her diabetes care, as previous report suggested the effectiveness of liraglutide in patients with FPLD1 for about three months. We assessed changes in fat distribution and insulin resistance during liraglutide therapy using dual energy X-ray absorptiometry (DEXA) and the oral glucose tolerance test (OGTT). Liraglutide effectively has achieved favorable glycemic and body weight control for a long period. The DEXA showed that fat mass in the upper limbs and trunk was increased, but we could speculate no further increase as her body weight was stable. The OGTT showed the amelioration of insulin resistance and insulin secretory capacity adjusted for insulin sensitivity. We thought that this might be caused by the blood-glucose-reducing effects of liraglutide and preventive effects on obesity through increasing the fat storage capacity of adipocytes. The intensified multifactorial intervention with liraglutide, anti-hypertensive drugs, and statin attenuated diabetic microangiopathies. The present case suggests that liraglutide might be as an efficient therapeutic option through its pleiotropic effects in diabetic patients with FPLD1.
\end{abstract}

\section{Introduction}

Lipodystrophy has been classified as inherited or acquired with the general or partial loss of adipose tissue deposits [1]. It is commonly associated with dyslipidemia, hepatic steatosis, and insulin-resistant diabetes. Familial partial lipodystrophy (FPLD) 1, also known as Köbberling type, is generally autosomal dominant; however, its genetic cause remains unknown. It is characterized by fat loss in the lower limbs with abdominal fat accumulation, a high rate of positivity for a family history, and metabolic disturbances [1-5]. Previous studies reported that FPLD1 is more common than considered in American and Japanese diabetic outpatient clinics [2,4,5]. Patients with FPLD1 frequently have insulin resistance due to abdominal fat accumulation and lipotoxicity in muscle and liver, caused by fat loss in the lower limbs. Therefore, they often have difficulty in maintaining good glycemic control and are predisposed to the deterioration of diabetic microangiopathies, including retinopathy and nephropathy. These patients are also predisposed to the development of severe atherosclerosis, caused by atherogenic risk factors, such as diabetes, dyslipidemia, and hypertension.

Type 2 diabetes is characterized by multiple pathophysiological disturbances, referred to as the ominous octet [6]. The glucagon-like peptide 1 (GLP-1) receptor agonist, liraglutide is used to treat 6 out of the 8 components, including the two major core defects, $\beta$-cell dysfunction and insulin resistance, in type 2 diabetics [7]. Liraglutide also exerts body weight-reducing effects through the suppression of gastrointestinal peristalsis and feeding central function in the hypothalamus and has been emphasized as an important drug for obese patients with type 2 diabetes $[7,8]$. Oliveira et al. was the first to show that GLP-1 receptor agonists effectively achieved favorable glycemic control for 3 or 4 months in two patients with partial lipodystrophy who appeared to fit the category of FPLD1 [9]. However, the long-term effects of GLP-1 receptor agonists on glycemic control and diabetic complications in patients with FPLD1 currently remain unclear.

The results of the present study indicated that the long-term administration of liraglutide effectively achieved glycemic and body weight control in a female Japanese diabetic patient. We performed dual energy X-ray absorptiometry (DEXA) and the oral glucose tolerance test (OGTT) twice during the liraglutide treatment to assess changes in fat distribution and insulin resistance, and the results obtained enabled

${ }^{*}$ Correspondence to: Masanori Iwanishi $\mathrm{PhD}, \mathrm{MD}$, Department of Diabetes and Endocrinology, Kusatsu General Hospital 1660 Yabase, Kusatsu, Shiga 525-8585, Japan, Tel: 077-563-8866; Fax: 077-563-0520; E-mail: masa-iwani@solid.ocn.ne.jp

Key words: partial lipodystrophy, familial partial lipodystrophy 1, a glucagon-like peptide-1 receptor agonist, liraglutide, diabetic microangiopathies

Received: December 12, 2020; Accepted: December 24, 2020; Published: January 05, 2021 
Iwanishi M (2021) Beneficial effects of long-term liraglutide monotherapy on glycemic control and diabetic microangiopathies in a female Japanese diabetic patient with familial partial lipodystrophy 1

us to speculate the mechanisms by which liraglutide may contribute to maintaining glycated hemoglobin $\mathrm{Alc}(\mathrm{HbAlc})$ reductions. Furthermore, we herein demonstrated that a long-term treatment with liraglutide, anti-hypertensive drugs, and statin may also have been beneficial for diabetic microangiopathies and macroangiopathy in this patient.

\section{Case report}

The present case was reported as case 5 of FPLD1 in our previous study [5]. Figure 1 and Figure 2 were reproduced from our previous study with permission [5].

\section{Patient profile and the clinical course of a diabetic foot ulcer}

A 50-year-old woman was admitted to our hospital with a right diabetic foot ulcer (Figure 1) for 3 weeks in Sep 2011 and was diagnosed with diabetes mellitus for the first time. On admission, her temperature was $37.2^{\circ} \mathrm{C}$, blood pressure $182 / 100 \mathrm{mmHg}$, weight $55.5 \mathrm{~kg}$, height 163 $\mathrm{cm}$, and body mass index (BMI) $20.9 \mathrm{~kg} / \mathrm{m}^{2}$. Seven years before her admission, her body weight was $65 \mathrm{~kg}$, which suggested BMI of $24.4 \mathrm{~kg} /$ $\mathrm{m}^{2}$. Thus, she had lost approximately $9 \mathrm{~kg}$ from seven years ago, although her oral intake was not inadequate. She had severe and moderate nonproliferative diabetic retinopathy in the right and left eyes, respectively (Figure 1). She also had diabetic nephropathy with microalbuminuria, but not diabetic neuropathy. Laboratory findings (Table 1) showed that her $\mathrm{HbA1c}$ level was 11.3\%, serum cholesterol level $182 \mathrm{mg} / \mathrm{dL}, \mathrm{WBC}$ $13,900 / \mu \mathrm{L}$, and CRP level $12.5 \mathrm{mg} / \mathrm{dL}$. She was a smoker (10 cigarettes a day for 30 years), but rarely drank alcohol. The right foot ulcer was treated with surgical debridement and antibiotics. Her diabetic foot ulcer completely healed one month after these treatments.

As shown in Figure 2, diabetic diet therapy of 1,600 Kcal and 3 units of novorapid immediately before each meal ( 9 units of insulin daily) were initiated. Five months after favorable glycemic control had been achieved, insulin was switched to liraglutide therapy (initially
$0.3 \mathrm{mg}$ and increased to $0.6 \mathrm{mg}$ daily). At her request, she continue liraglutide therapy without any other hypoglycemic drugs. Liraglutide therapy achieved favorable glycemic control for 7 years. As shown in Figure 3, telmisartan $40 \mathrm{mg}$ and azelnidipine $16 \mathrm{mg}$ were administered for hypertension in Sep. 2011, followed by pravastatin $10 \mathrm{mg}$ to prevent atherosclerosis in Mar. 2012. Blood pressure was controlled at 120/70 $\mathrm{mmHg}$ and her serum cholesterol level has remained within normal ranges (Table 1).

\section{Changes in fat distribution, insulin resistance, and glycemic control during liraglutide therapy in this patient}

We examined the body composition of our patient using a DEXA scan twice (Sep. 2011 and Oct. 2018). As shown in Table 2, the body

Table 1. Laboratory data for our patient

\begin{tabular}{|c|c|c|c|}
\hline Time & Aug.2011 & Jun.2012 & \\
\hline Metabolic variables & & & Normal Values \\
\hline HbA1c $(\%)$ & 11.3 & 6.5 & $4.6-6.2$ \\
\hline Plasma glucose $(\mathrm{mg} / \mathrm{dL})$ & 314 & 127 & $70-109$ \\
\hline Plasma insulin $(\mu \mathrm{U} / \mathrm{mL})$ & ND & 12.8 & $2.7-10.4$ \\
\hline Serum cholesterol $(\mathrm{mg} / \mathrm{dL})$ & 182 & 177 & $130-219$ \\
\hline Serum triglyceride $(\mathrm{mg} / \mathrm{dL})$ & ND & 79 & $30-149$ \\
\hline Serum HDL cholesterol $(\mathrm{mg} / \mathrm{dL})$ & ND & 46 & $40-90$ \\
\hline Serum leptin $(\mathrm{ng} / \mathrm{mL})$ & ND & 4.9 & \\
\hline Serum adiponectin $(\mu \mathrm{g} / \mathrm{mL})$ & ND & 4.1 & \\
\hline WBC $\left(10^{2} / \mu \mathrm{L}\right)$ & 139 & 74 & $35-91$ \\
\hline Hb $(\mathrm{g} / \mathrm{dL})$ & 12.7 & 12.1 & $11.3-15.2$ \\
\hline PLTs $\left(10^{4} / \mu \mathrm{L}\right)$ & 31.8 & 20.4 & $13.0-36.9$ \\
\hline $\mathrm{CRP}(\mathrm{mg} / \mathrm{dL})$ & 12.5 & ND & $0.3 \geq$ \\
\hline $\mathrm{AST}(\mathrm{U} / \mathrm{L})$ & 8 & 19 & $8-38$ \\
\hline $\mathrm{ALT}(\mathrm{U} / \mathrm{L})$ & 8 & 30 & $4-43$ \\
\hline $\mathrm{LDH}(\mathrm{U} / \mathrm{L})$ & 136 & 165 & $121-245$ \\
\hline
\end{tabular}

Blood sample on Aug. 2011 was obtained after breakfast, while blood sample on Jun. 2012 was obtained after a 12-hour overnight fast.
A DO: Fat atrophy

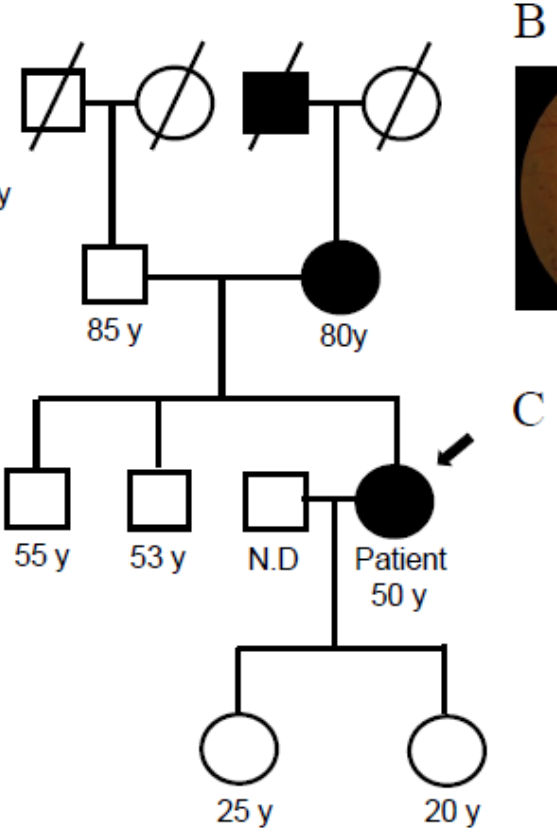

B
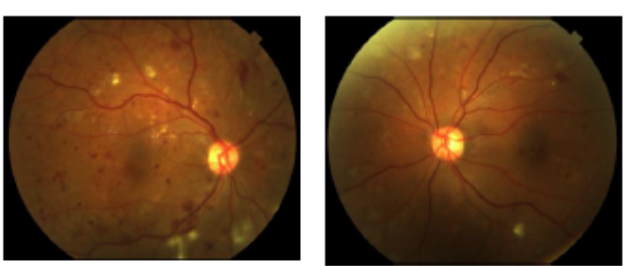

$\mathrm{C}$

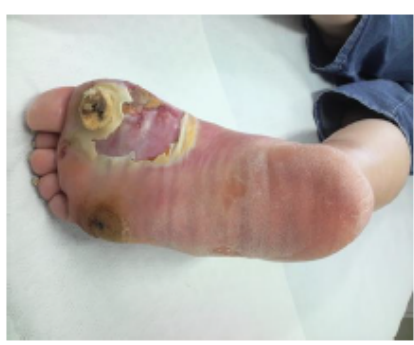

Figure 1. (A)The family pedigree of this patient. The proband is denoted by an arrow (B) Images taken in the first fundoscopic examination by an ophthalmologist on 31 Aug. 2011 (C) The right diabetic foot ulcer at her first visit was shown. Figure 1C was reproduced from our previous study with permission [5] 
Iwanishi M (2021) Beneficial effects of long-term liraglutide monotherapy on glycemic control and diabetic microangiopathies in a female Japanese diabetic patient with familial partial lipodystrophy 1

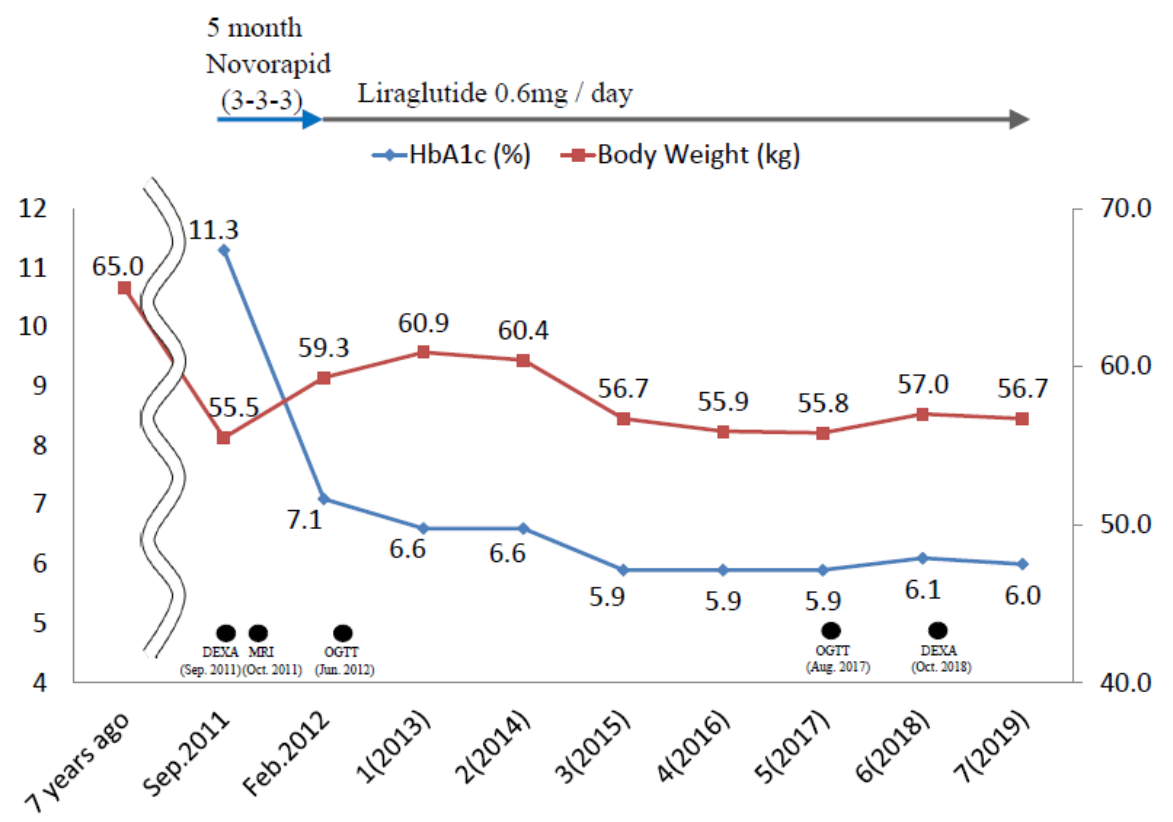

Figure 2. Clinical course and changes in glycated hemoglobin (HbA1c) levels and body weight between 2011 and 2019. Body weight was $55.5 \mathrm{~kg}$ in Sep. 2011 . Seven years before her admission, body weight was $65 \mathrm{~kg}$. After insulin had been administered for 5 months, her HbAlc level decreased to $7.1 \%$ from $11.3 \%$ on admission and body weight gain of approximately $4 \mathrm{~kg}$ was observed 3 months after the initiation of insulin. After switching to $0.6 \mathrm{mg}$ liraglutide in Feb. 2012, she achieved a favorable HbA1 $\mathrm{c}$ level and body weight fluctuated between 56 and $57 \mathrm{~kg}$. DEXA was performed in Sep. 2011 and Oct. 2018 and MRI in Oct. 2011, while OGTT was performed in Jun. 2012 and Aug. 2018

A

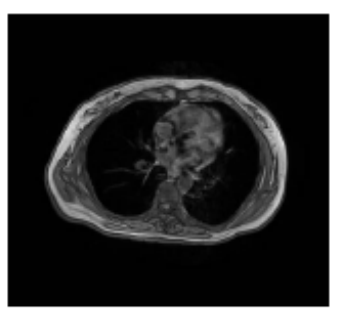

B

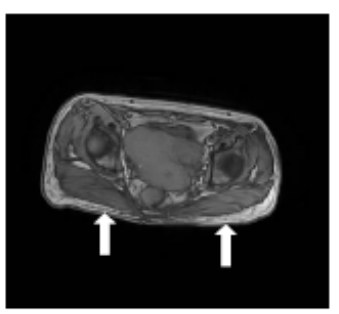

C

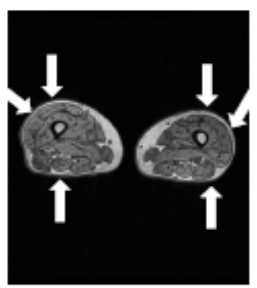

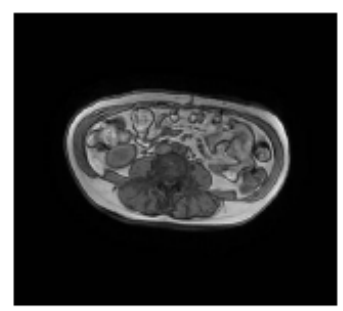

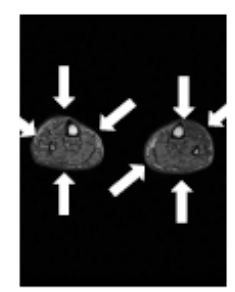

$\mathrm{D}$

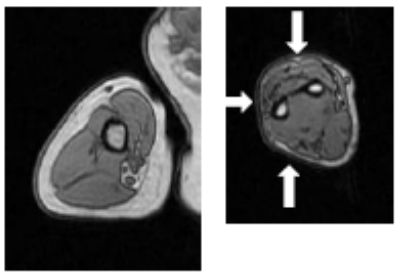

$\mathrm{E}$

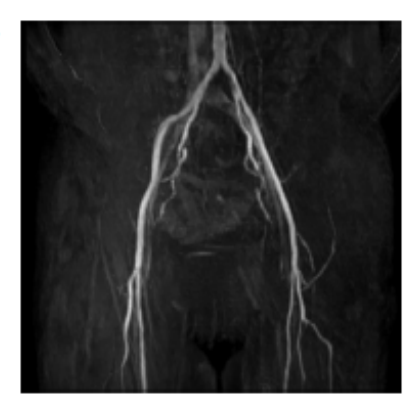

Figure 3. MRI in body was performed in Oct. 2011 when her body weight was $55.5 \mathrm{~kg}$ (A) Thoracic MRI taken at the level of the seventh thoracic vertebrae (left panel) and abdominal MRI at the umbilical level (right panel) showed the preservation of subcutaneous fat in thoracic and abdominal regions (B) T1-weighted MRI taken at the level of gluteal fat indicated a decrease in gluteal subcutaneous fat, as shown by arrows (C) MRI taken at the level of the thigh (left panel), calf (middle panel), and left leg (right panel) were shown. The patient had very little subcutaneous fat, particularly in the antero-lateral and posterior thigh regions, indicated by arrows, whereas subcutaneous fat in the internal thigh region remained. She had the near-complete loss of subcutaneous fat in the entire circumference of the calf, as indicated by arrows. She had the almost complete absence of dorsal and plantar subcutaneous fat, as indicated by arrows (D) MRI taken at the level of the right arm (left panel) and forearm (right panel) revealed the preservation of subcutaneous fat and the almost complete absence of subcutaneous fat in the antero-lateral and posterior forearm regions, as indicated by arrows; however, small amounts of subcutaneous fat in the internal forearm were preserved (E) Magnetic resonance angiography (MRA) of the lower limbs was performed in Jul. 2019. Figure A-D were reproduced from our previous study with permission [5] 
Iwanishi M (2021) Beneficial effects of long-term liraglutide monotherapy on glycemic control and diabetic microangiopathies in a female Japanese diabetic patient with familial partial lipodystrophy 1

composition of this patient on DEXA was compared with those in BMI- and age-matched groups (Controls 1 and 2 groups, respectively). Control 1 group with 56 healthy female volunteers aged between 40 and 49 years were recruited from the employees of Tanita, Inc. Control 2 group with 41 healthy female volunteers aged between 50 and 59 years was also recruited from the employees of Tanita. Inc. The mean ages of Control groups 1 and 2 were 45.1 and 52.8 years, respectively, while mean BMI were 22.7 and $23.5 \mathrm{~kg} / \mathrm{m}^{2}$, respectively. Truncal fat mass in DEXA in Sep. 2011 in our patient was higher than those in Control groups 1 and 2, while lower limb fat mass was lower than those in Control groups 1 and 2. DEXA in Oct. 2018 revealed that her body weight had increased by approximately $1.5 \mathrm{~kg}$ due to favorable glycemic control. Total fat mass in DEXA in Oct. 2018 was $4.1 \mathrm{~kg}$ higher than that in DEXA in Sep. 2011, whereas total lean mass was $2.2 \mathrm{~kg}$ lower. Truncal fat mass was higher and fat loss was greater in the lower limbs in DEXA in Oct. 2018 than in Control groups 1 and 2. Lower limb fat mass in DEXA in Oct. 2018 was $3.9 \mathrm{~kg}$, being less than the mean -2SD of that of Control group 1. This result indicated how fat is distributed in patients with FPLD1. We previously reported that the fat mass ratio (FMR) is useful for diagnosing FPLD 1 [4]. FMR is the ratio of the percentage of trunk fat mass to that of lower limb fat mass. FMR was 1.2 in two DEXA in this patient, while mean FMR in Control 1 group was 1.0. Figure 3 shows magnetic resonance imaging (MRI) when her body weight was $55.5 \mathrm{~kg}$. Fat loss was observed in the lower limbs with the preservation of subcutaneous fat in thoracic and abdominal regions.

We also performed OGTT on our patient in Jun. 2012 and Aug. 2017 and assessed glucose tolerance, insulin secretion, and insulin resistance. We presented the values for the homeostasis model assessment of insulin resistance (HOMA-IR), homeostasis model assessment of $\beta$-cell function (HOMA- $\beta$ ), Matsuda index, disposition index, and insulinogenic index in 17 normoglycemic subjects with a mean age of 53.3 years and mean BMI of $23.6 \mathrm{~kg} / \mathrm{m}^{2}$ in Table 3 [1012]. We converted plasma glucose and plasma insulin from $\mathrm{mmol} / \mathrm{L}$ and $\mathrm{pmol} / \mathrm{L}$ to $\mathrm{mg} / \mathrm{dL}$ and $\mu \mathrm{U} / \mathrm{ml}$. Based on these values, we converted the values in disposition index and insulinogenic index drawn from reference 10 to the values, which we presented in Table 3. Regarding the insulin secretory capacity, the two sets of results obtained in OGTT showed a lower HOMA- $\beta$, disposition index, and insulinogenic index than those in normal subjects. Concerning insulin resistance, HOMA-IR was 4.0 and the Matsuda index was 2.0 in OGTT in Jun. 2012; HOMA-IR was higher, while the Matsuda index was lower than those in normal subjects. Yamada et al. showed that HOMA-IR $\geq 2.5$ indicates insulin resistance in Japanese individuals [13], while Takahara et al. reported that a Matsuda index $<4.3$ suggests insulin resistance in Japanese individuals [14]. The values obtained for our patient indicated the presence of insulin resistance. Therefore, we found insulin resistance in addition to an insulin secretory defect in this patient. In contrast, OGTT in Aug. 2017 showed a lower HOMA-IR and higher Matsuda index than those in OGTT in Jun. 2012, but hyperinsulinemia at 2 hour. Thus, this result suggested the amelioration of, but still persistent, insulin resistance. In addition, it showed higher disposition index, suggesting that an insulin secretory capacity adjusted for insulin sensitivity was improved.

Whole-exome sequencing has been performed to examine whether patients have mutations in candidate genes that are associated with FPLD, including LMNA, PPARG, PLIN1, CIDEC, LIPE, ADRA2, and AKT2 $[4,5]$. Our patient did not have mutations in the candidate genes associated with FPLD or any novel candidate causal genes. We showed the family pedigree of this patient based on an interview (Figure 1). Her mother and grandfather had diabetes with fat loss in the lower limbs, suggesting an autosomal dominant pattern of inheritance. She noted fat loss in the lower limbs during adolescence. Therefore, she appeared to fit the category of FPLD1. However, we were unable to perform genetic analyses or obtain any clinical data on any other family members.

\section{Diabetic microangiopathies and macroangiopathy}

As shown in Figure 1, images taken in the first fundoscopic examination by an ophthalmologist showed microaneurysms, hard exudates, cotton-wool spots, and intra-retinal hemorrhage in the four quadrants of her right eye, suggesting severe non-proliferative diabetic retinopathy, as well as microaneurysms, hard exudates, cotton-wool spots, and intra-retinal hemorrhage in fewer than the four quadrants in

Table 2. Body composition of this patient in DEXA

\begin{tabular}{|c|c|c|c|c|}
\hline Time & 2011 September & 2018 October & Control $1(n=56)$ & Control $2(n=41)$ \\
\hline Gender & Female & Female & Female & Female \\
\hline Age (y) & 50 & 57 & $45.1 \pm 2.8$ & $55.2 \pm 3.1$ \\
\hline Height $(\mathrm{cm})$ & 163.0 & 163.0 & $156.1 \pm 4.9$ & $156.8 \pm 4.7$ \\
\hline Body weight (kg) & 55.5 & 57.0 & $55.1 \pm 6.7$ & $55.1 \pm 6.3$ \\
\hline BMI $\left(\mathrm{kg} / \mathrm{m}^{2}\right)$ & 20.9 & 21.5 & $22.7 \pm 3.0$ & $23.5 \pm 2.9$ \\
\hline Fat $(\%)$ Total $\%$ Fat & 25.2 & 31.8 & $30.3 \pm 6.2$ & $31.4 \pm 6.5$ \\
\hline Upper Limbs \% Fat & 29.1 & 39.1 & $27.6 \pm 7.1$ & $29.6 \pm 7.8$ \\
\hline Trunk \% Fat & 26.1 & 33.8 & $28.4 \pm 7.7$ & $31.2 \pm 7.6$ \\
\hline Lower Limbs \% Fat & 22.0 & 28.1 & $33.2 \pm 5.7$ & $32.5 \pm 6.2$ \\
\hline Fat Mass (kg) Total Fat Mass & 13.7 & 17.8 & $16.8 \pm 5.2$ & $17.3 \pm 5.3$ \\
\hline Upper Limbs Fat Mass & 1.6 & 2.4 & $1.4 \pm 0.3$ & $1.6 \pm 0.3$ \\
\hline Trunk Fat Mass & 7.9 & 10.6 & $7.1 \pm 2.8$ & $8.0 \pm 2.9$ \\
\hline Lower Limbs Fat Mass & 3.5 & 3.9 & $7.0 \pm 1.0$ & $6.5 \pm 0.9$ \\
\hline Lean Mass (kg) Total Lean Mass & 38.9 & 36.7 & $35.3 \pm 3.1$ & $34.6 \pm 3.0$ \\
\hline Upper Limbs Lean Mass & 3.5 & 3.6 & $3.4 \pm 0.2$ & $3.4 \pm 0.3$ \\
\hline Trunk Lean Mass & 21.8 & 20.4 & $16.3 \pm 1.3$ & $16.3 \pm 1.7$ \\
\hline Lower Limbs Lean Mass & 10.4 & 9.7 & $13.1 \pm 0.9$ & $12.3 \pm 0.7$ \\
\hline Fat mass Ratio (FMR) & 1.2 & 1.2 & $1.0 \pm 0.3$ & \\
\hline
\end{tabular}

Normal values in Control 1 were obtained from 56 healthy women between the ages of 40 and 49 years.

Normal values in Control 2 were obtained from 41 healthy women between the ages of 50 and 59 years.

Values in Control 1 and Control 2 were expressed as mean \pm SD. 
Iwanishi M (2021) Beneficial effects of long-term liraglutide monotherapy on glycemic control and diabetic microangiopathies in a female Japanese diabetic patient with familial partial lipodystrophy 1

her left eye, indicating moderate non-proliferative diabetic retinopathy. Corrected vision in the right and left eyes were $6 / 8$ and $6 / 7$, respectively. Since she had severe diabetic retinopathy, she received regular eye examinations. Fluorescein angiography was performed one week after the fundoscopic examination. It showed a broad non-perfused area in the right retina, on which laser photocoagulation was performed. One year after the initiation of diabetic medication, fluorescein angiography showed that the non-perfused area had increased in her left retina; therefore, laser photocoagulation was also performed on the left retina in 2012. As shown in Figure 4, optical coherence tomography (OCT) initially did not show any diabetic macular edema (DME). However, DME was detected in the right and left eyes in Jan. 2013 and Mar. 2013, respectively. Laser photocoagulation was subsequently performed around the right macula, resulting in a decrease in vision to 6/20. DME was resolved in both eyes in 2014 after her glucose profile had been stabilized. She underwent vitrectomy for vitreous hemorrhage of the left fundus in 2016. As shown in Figure 4, her fundus was stable in the most recent fundoscopic examination conducted by the ophthalmologist in Feb. 2020, and final corrected vision in the right and left eyes were $6 / 20$ and $6 / 6$, respectively.

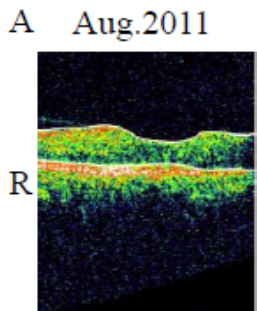

Jan. 2013

Mar.2013

Jun. 2013

Apr. 2014

Apr. 2018
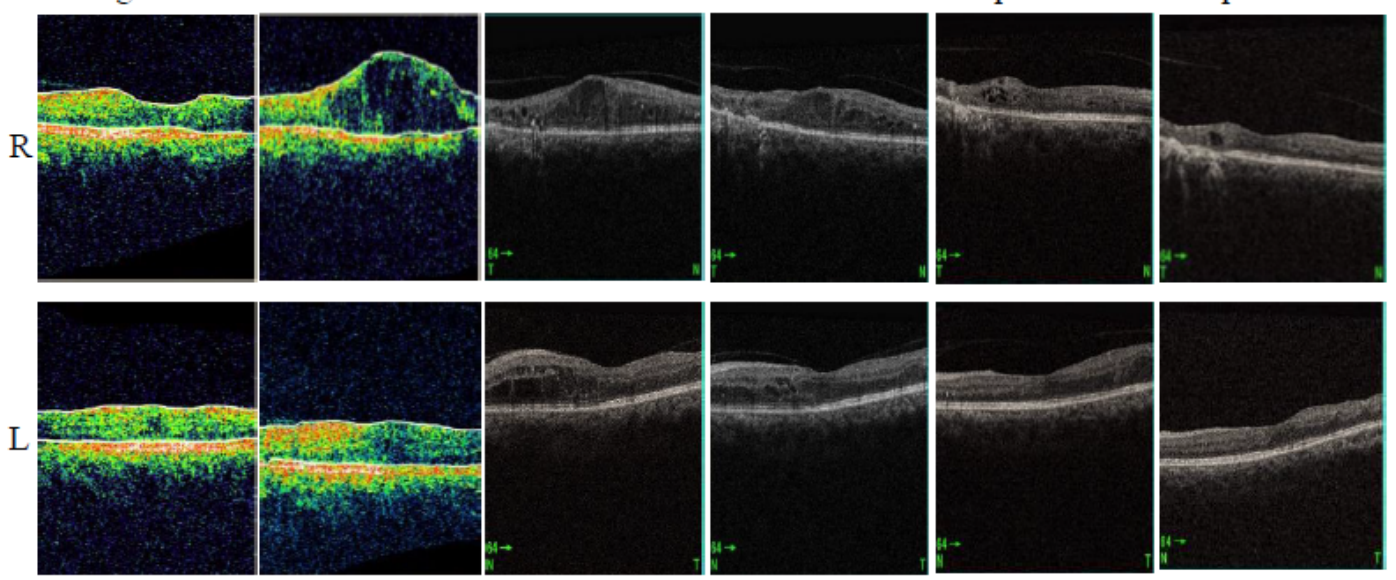

B
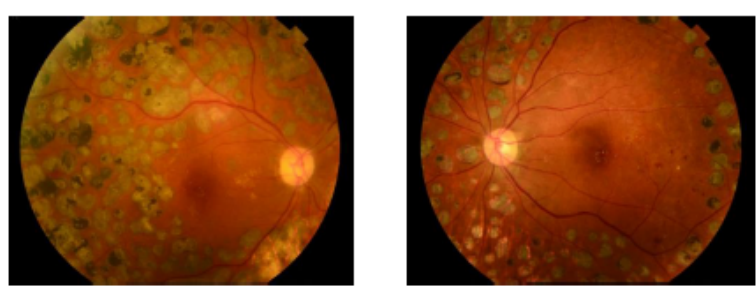

Figure 4. (A) Optical coherence tomography (OCT) images showing chronological changes from 2011 to 2018. OCT images in Aug. 2011 and Jan. 2013 were obtained by Carl Zeiss OCT3000, and in Mar. 2013, Jun. 2013, Apr. 2014, and Apr. 2018 by TOPCON OCT-2000. OCT in the first examination by the ophthalmologist in Aug. 2011 showed no diabetic macular edema (DME). The OCT image in Jan. 2013 showed the deterioration of DME in the right eye. Approximately 1.5 years after the initiation of diabetic medication, the OCT image in Mar. 2013 showed the deterioration of DME in the left eye. However, since blood glucose levels had been improving, the OCT image showed the attenuation of DME in both eyes since 2014 (B) When fundoscopic photography was performed in the most recent examination by the ophthalmologist in Feb. 2020, her fundus was stable and corrected vision in right and left eyes were $6 / 20$ and $6 / 6$, respectively, suggesting the preservation of vision

Table 3. Oral glucose tolerance test and profile of insulin secretion and insulin resistance in this patient

\begin{tabular}{|c|c|c|c|c|c|c|c|c|c|c|c|c|c|c|c|}
\hline \multirow[b]{2}{*}{ Time } & \multirow{2}{*}{$\begin{array}{l}\text { BW } \\
(\mathrm{kg})\end{array}$} & \multirow{2}{*}{$\begin{array}{c}\text { BMI } \\
\left(\mathrm{kg} / \mathrm{m}^{2}\right) \\
\text { at OGTT }\end{array}$} & \multirow{2}{*}{$\begin{array}{c}\text { HbA1c } \\
(\%)\end{array}$} & & & & \multicolumn{4}{|c|}{ Time(min) } & \multirow[b]{2}{*}{ HOMA-IR } & \multirow[b]{2}{*}{ НОМА- } & \multirow{2}{*}{$\begin{array}{l}\text { Matsuda } \\
\text { Index }\end{array}$} & \multirow{2}{*}{$\begin{array}{l}\text { Disposition } \\
\text { Index }\end{array}$} & \multirow{2}{*}{$\begin{array}{l}\text { Insulinogenic } \\
\text { Index }\end{array}$} \\
\hline & & & & & & & 0 & 30 & 60 & 120 & & & & & \\
\hline \multirow{2}{*}{$\begin{array}{l}\text { JUN } \\
2012\end{array}$} & \multirow{2}{*}{60.5} & \multirow{2}{*}{22.8} & \multirow{2}{*}{6.6} & \multirow{2}{*}{\multicolumn{3}{|c|}{$\begin{array}{l}\text { Plasma glucose }(\mathrm{mg} / \mathrm{dL}) \\
\text { Plasma insulin }(\mu \mathrm{U} / \mathrm{mL})\end{array}$}} & 127.0 & 179.0 & 249.0 & 262.0 & \multirow{2}{*}{4.0} & \multirow{2}{*}{72.0} & \multirow{2}{*}{2.0} & \multirow{2}{*}{0.6} & \multirow{2}{*}{0.28} \\
\hline & & & & & & & 12.8 & 27.3 & 54.2 & 157.6 & & & & & \\
\hline \multirow{2}{*}{$\begin{array}{c}\text { August } \\
2017\end{array}$} & \multirow{2}{*}{55.8} & \multirow{2}{*}{21.1} & \multirow{2}{*}{6.0} & & ma gluco & $(\mathrm{mg} / \mathrm{dL})$ & 110.0 & 178.0 & 206.0 & 217.0 & \multirow{2}{*}{2.2} & \multirow{2}{*}{62.0} & \multirow{2}{*}{3.4} & \multirow{2}{*}{0.9} & \multirow{2}{*}{0.25} \\
\hline & & & & \multicolumn{3}{|c|}{ Plasma insulin $(\mu \mathrm{U} / \mathrm{mL})$} & 8.1 & 25.3 & 34.4 & 123.3 & & & & & \\
\hline \multirow{2}{*}{\multicolumn{4}{|c|}{$\begin{array}{c}\text { The values in } \\
\text { normoglycemic subjects }\end{array}$}} & $\mathrm{n}$ & $\begin{array}{l}\text { Age } \\
\text { (years) }\end{array}$ & $\begin{array}{c}\text { BMI } \\
\left(\mathrm{kg} / \mathrm{m}^{2}\right) \\
\text { at OGTT }\end{array}$ & & & & & \multirow{2}{*}{$\begin{array}{c}1.92 \\
(1.25)\end{array}$} & \multirow{2}{*}{$\begin{array}{c}78.3 \\
(46.1)\end{array}$} & \multirow{2}{*}{$\begin{array}{c}5.62 \\
(2.87)\end{array}$} & \multirow{2}{*}{$\begin{array}{c}1.61 \\
(0.54)\end{array}$} & \multirow{2}{*}{$\begin{array}{c}0.62 \\
(0.48)\end{array}$} \\
\hline & & & & 17 & $\begin{array}{l}53.3 \\
(3.3)\end{array}$ & $\begin{array}{l}23.6 \\
(2.7)\end{array}$ & & & & & & & & & \\
\hline
\end{tabular}

BW: Body Weight OGTT: Oral Glucose Tolerance Test.

HOMA-IR: Homeostasis model assessment of insulin resistance.

HOMA- $\beta$ : Homeostasis model assessment of $\beta$-cell function.

The Mean \pm SD in normoglycemic subjects are in the bottom. The figures in parentheses are SD

These values were drawn from Reference [10]. 
Iwanishi M (2021) Beneficial effects of long-term liraglutide monotherapy on glycemic control and diabetic microangiopathies in a female Japanese diabetic patient with familial partial lipodystrophy 1

Figure 5 shows the clinical course of the estimated glomerular filtration rate (eGFR) and excretion of urinary albumin. She initially exhibited glomerular hyperfiltration and increased microalbuminuria. However, during an intensified multifactorial intervention, both parameters of diabetic nephropathy gradually decreased to normal levels.

Regarding atherosclerosis, the right and left ankle brachial pressure indexes (ABI) on admission were 0.95 and 0.87 , respectively; however, both ABI subsequently improved and stabilized to within normal ranges. As shown in Figure 3, bilateral lower limb magnetic resonance angiography (MRA) performed in 2019 showed no narrowing of the arteries from the aorta to the bilateral popliteal region. Images of the right posterior tibial artery (PTA), left anterior tibial artery (ATA), and left PTA were unclear. Bilateral carotid arterial echography performed in 2011 on this patient showed no atherosclerotic lesions. However, we were unable to assess the degree of atherosclerosis in coronary arteries and cerebral arteries because the patient refused to undergo multidetector-row computed tomography (MDCT) containing 64 slices or brain MRA.

\section{Discussion}

We herein assessed changes in fat distribution and insulin resistance during liraglutide therapy using DEXA and OGTT. In the first visit, body weight was $55.5 \mathrm{~kg}$. The patient had lost approximately $9 \mathrm{~kg}$ from seven years ago, although her oral intake was not inadequate. Retinopathy showed severe non-proliferative diabetic retinopathy and her HbAlc level was $11.0 \%$. Therefore, she had poor glycemic control for a long period and body weight reductions with decreased fat mass had been caused by uncontrolled diabetes [15]. Her body weight increased by approximately $1.5 \mathrm{~kg}$ in DEXA in Oct. 2018 after achieving favorable glycemic control. Truncal fat mass and upper limb fat mass were higher than in DEXA in Sep. 2011. In contrast, lower limb fat mass only slightly increased. This result supported previous findings showing that preadipocytes differentiate less efficiently to mature adipocytes due to a genetic defect in adipocyte differentiation in lipodystrophic areas $[16,17]$. OGTT revealed insulin resistance. We previously reported that the cut-off point of FMR to diagnose lipodystrophy is 1.2 (sensitivity $100 \%$, specificity $91 \%$ ) [4]. Since FMR in this patient was 1.2 , we suspected lipodystrophy. Thus, the present results appear to support the diagnosis of partial lipodystrophy in our patient.

The majority of diabetic patients with FPLD1 have multiple pathophysiological disturbances, referred to as the ominous octet in type 2 diabetes. We found that the long-term administration of liraglutide effectively achieved favorable glycemic control in this patient. The three main factors that contributed to this result, namely, the amelioration of insulin resistance and an insulin secretory defect as well as other factors, are important.

The attenuation of insulin resistance is a crucial factor, to which favorable glycemic control may contribute. This patient had uncontrolled diabetes with a decreased fat mass in her first visit. Total fat mass was $4.1 \mathrm{~kg}$ higher in DEXA in Oct. 2018 than in DEXA in Sep.

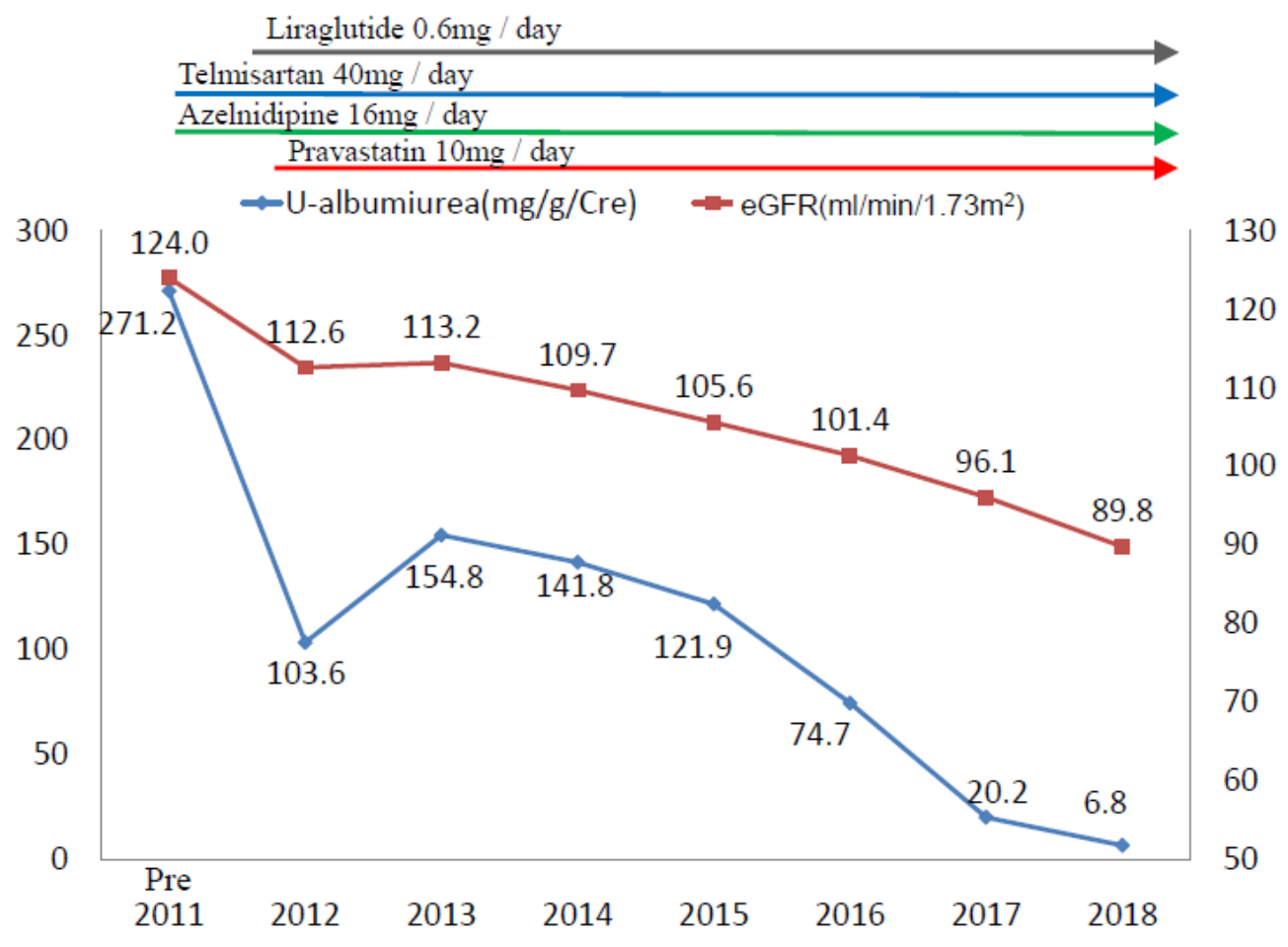

Figure 5. Changes in albuminuria $(\mathrm{mg} / \mathrm{g} / \mathrm{Cre})$ and eGFR $\left(\mathrm{mL} / \mathrm{min} / 1.73 \mathrm{~m}^{2}\right)$ between 2011 and 2018 . Urinary albumin levels in individual years were the mean values of two random urine samples. Liraglutide $0.6 \mathrm{mg}$ was administered in Feb. 2012. Telmisartan $40 \mathrm{mg}$ and azelnidipine $16 \mathrm{mg}$ were administered for hypertension in Sep. 2011 and pravastatin $10 \mathrm{mg}$ for the prevention of atherosclerosis in Mar. 2012. Her urinary albumin level on admission was $271.2 \mathrm{mg} / \mathrm{g} / \mathrm{Cre}$, suggesting that she had diabetic nephropathy with microalbuminuria. eGFR on admission was $124.0 \mathrm{~mL} / \mathrm{min} / 1.73 \mathrm{~m}^{2}$, suggesting hyperfiltration in the glomerulus. Since her blood glucose level was improving, her urinary albumin level gradually decreased to the normal range and a reduction in eGFR to $89.8 \mathrm{~mL} / \mathrm{min} / 1.73 \mathrm{~m}^{2}$ was also observed 
Iwanishi M (2021) Beneficial effects of long-term liraglutide monotherapy on glycemic control and diabetic microangiopathies in a female Japanese diabetic patient with familial partial lipodystrophy 1

2011, whereas total lean mass was $2.2 \mathrm{~kg}$ lower. Truncal fat mass and upper limb fat mass in DEXA in Oct. 2018 following favorable glycemic control were larger than in DEXA in Sep. 2011. The increase observed in fat mass may be associated with the maintenance of favorable glycemic control. Based on changes in body composition assessed by DEXA and $\mathrm{CT}$, liraglutide reduces fat mass rather than lean mass and decreases the visceral adipose tissue area more than the subcutaneous area in patients with type 2 diabetes $[18,19]$. Changes in fat mass in our patient were incompatible with these findings and may be attributed to the change from long-term uncontrolled diabetes to controlled diabetes. Under poor long-term glycemic control, weight loss, a decrease in fat mass, malnutrition, and dehydration are reported in many cases. Glucose utilization efficiency is low and, thus, fat is consumed to obtain energy; therefore, lipolysis is promoted in adipose tissue. In patients with hyperglycemia, the fat storage capacity of adipocytes decreases due to enhanced oxidative stress and the exacerbation of inflammation [20,21]. Furthermore, the differentiation capacity from preadipocytes to mature adipocytes decreases, thereby reducing the number of adipocytes. Thus, the decreased fat mass associated with uncontrolled diabetes may be attributed to a reduced fat storage capacity in adipocytes with smaller fat cell numbers. Therefore, the maintenance of glycemic control by liraglutide may increase the fat storage capacity of adipocytes through the prevention of oxidative stress, amelioration of inflammation, and increases in fat cell numbers by enhancing the differentiation capacity of mature adipocytes from preadipocytes. These results suggest that the maintenance of glycemic control by liraglutide causes healthy adipose expansion from unhealthy adipose expansion in adipocytes [22,23]. Improved fat cell function may decrease ectopic lipid deposition in the liver and muscle through fat loss in the lower limbs, resulting in the attenuation of insulin resistance [24].

The prevention of obesity, particularly excess truncal fat, may have also contributed to the attenuation of insulin resistance in our patient. Patients with FPLD1 often have difficulty in maintaining good glycemic control with classical therapeutic options. Pinnick et al. previously reported that android fat mass was positively associated with insulin resistance, while gynoid fat mass was negatively associated with insulin resistance [25]. They also showed that based on an analysis of transcriptional genes, abdominal subcutaneous fat was associated with the stronger expression of proinflammatory cytokines, which are associated with insulin resistance. Furthermore, Melvin et al. demonstrated that metabolic surgery was effective for glycemic control in patients with FPLD1 [26]. They suggested that this may have been due to the attenuation of insulin resistance after a decrease in truncal fat mass. Thus, truncal fat appears to play an important role in insulin resistance in patients with FPLD1. Since liraglutide exerts body weight-reducing effects with decreases in fat $[18,19]$, it may prevent excess truncal fat and inhibit severely hypertrophied adipocytes with unhealthy adipose expansion [22,23]. This appears to be one of the main mechanisms by which liraglutide maintained favorable glycemic control in our patient. Previous studies reported that liraglutide itself may increase the fat storage capacity by facilitating adipogenesis or increasing insulin sensitivity [27-29]. Therefore, liraglutide appears to ameliorate insulin resistance by increasing the fat storage capacity of adipocytes through liraglutide-induced favorable glycemic control, preventing obesity, particularly excess truncal fat, and its direct actions on adipocytes, thereby maintaining favorable glycemic control.

The attenuation of an insulin secretory defect appears be another important factor. The disposition index is considered to be a useful measure of $\beta$-cell function, reflecting insulin secretion capacity adjusted for insulin sensitivity [10]. The results of OGTT in Jun. 2012 and Aug. 2018 showed a lower HOMA- $\beta$, disposition index, and insulinogenic index than those in normal subjects. Thus, an insulin secretory defect was detected in our patient. Since liraglutide has been shown to promote early insulin secretion in patients with type 2 diabetes and those with FPLD2 [30,31], we speculated that this effect may also contribute to the maintenance of favorable glycemic control in our patient. Although the disposition index increased from 0.6 to 0.9 , no marked change was observed in the insulinogenic index between the two OGTT. Thus, this increase in the disposition index may suggest that an insulin secretory capacity adjusted for insulin sensitivity was improved, which mainly caused by the amelioration of insulin resistance.

The other effects of liraglutide are also an important factor. Liraglutide maintains favorable glycemic control by inhibiting the secretion of glucagon, reducing hepatic glucose production, or slowing the absorption of ingested glucose due to delayed gastric emptying.

We showed that the long-term administration of liraglutide with anti-hypertensive drugs and statin was effective against diabetic retinopathy and nephropathy. The results of MRA suggested the absence of severe atherosclerotic lesions in these arteries. Therefore, these drugs may have prevented the development of severe atherosclerotic lesions in the lower limbs. Severe non-proliferative diabetic retinopathy was detected in the first fundoscopic examination conducted by the ophthalmologist. DME deteriorated after the diabetic treatment. A reduced blood supply in the left eye and DME in both eyes after the diabetic treatment were considered to reflect the initial deterioration of diabetic retinopathy after favorable glycemic control had been achieved [32]. Laser photocoagulation was performed in the area around the right macula; however, corrected vision in the right eye decreased to $6 / 20$. The patient underwent vitrectomy for vitreous hemorrhage of the left fundus with a good outcome and no decrease in vision. We did not administer an anti-vascular endothelial growth factor (VEGF) antibody to treat DME in this patient because it had not yet been approved in Japan in Jan. 2013. Despite timely photocoagulation and medical treatments that led to a stable fundus, vision in the right eye decreased. Therefore, better therapeutic strategies need to be developed for diabetic patients with non-proliferative severe diabetic retinopathy [33].

The maintenance of favorable glycemic control was previously shown to be protective against diabetic retinopathy, diabetic nephropathy, and diabetic macroangiopathy [34-37]. Statin and angiotensin II receptor blockade (ARB) also exerted protective effects against diabetic retinopathy, diabetic nephropathy, and diabetic macroangiopathy [34-36,38]. Liraglutide induces reno-protection through its antioxidant effects in the endothelial cells of the glomerulus [39]. Although it is unclear whether these findings may be generalized to our patient who received $0.6 \mathrm{mg}$ liraglutide, $1.8 \mathrm{mg}$ of liraglutide has been reported to exert reno-protective effects and may effectively prevent the development of atherosclerosis in patients with type 2 diabetes $[40,41]$. Since liraglutide has been shown to increase cAMP levels through GLP-1 receptors in macrophages, thereby inhibiting the expression of proinflammatory cytokines, we speculated that the antiinflammatory effects of liraglutide on macrophages may have slowed the atherosclerotic process in the lower limbs of our patient $[42,43]$.

Xing et al. reported that patients with type 2 diabetes treated with liraglutide had less glycemic variability than those not treated with liraglutide in addition to milder hypoglycemia based on a meta-analysis of the literature [44]. We speculated that less glycemic 
Iwanishi M (2021) Beneficial effects of long-term liraglutide monotherapy on glycemic control and diabetic microangiopathies in a female Japanese diabetic patient with familial partial lipodystrophy 1

variability contributed to improvements in fat cell function, diabetic microangiopathy, and macroangiopathy through the prevention of oxidative stress in our patient [45]. Therefore, the attenuation of diabetic retinopathy and nephropathy and the stabilization of atherosclerosis in the lower limbs of our patient may have been due to the intensified multifactorial intervention with these drugs $[35,36]$.

In conclusion, this patient had fat loss in the lower limbs. She had insulin secretory defect and relatively severe insulin resistance. The long-term administration of liraglutide may have been effective for glycemic and body weight control. This might be caused by the blood-glucose-reducing effects of liraglutide, preventive effects on obesity. An intensified multifactorial intervention with liraglutide, antihypertensive drugs, and statin may have contributed to the amelioration of diabetic complications.

The present case suggests that liraglutide might be an efficient therapeutic option through its pleiotropic effects in female Japanese diabetic patients with FPLD1.

\section{Ethical consideration}

The present study (approved on 2 Sep. 2016, approval number: 2016090203), including whole-exome sequencing (approved on 12 Dec. 2016, approval number: 2016120206), was approved by the Ethics Committee of Kusatsu General Hospital. The patient agreed to participate and provided her written informed consent.

\section{Disclosure}

The authors state that they have no conflicts of interest (COI).

\section{References}

1. Akinci B, Sahinoz M, Oral E (2018) Lipodystrophy syndrome: Presentation and treatment endotext [Internet]. South Dartmouth (MA): MDText.com, Inc 2000 Apr 24.

2. Herbst KI, Purnell JQ, Tannock LR (2003) Köbberling type of familial partial lipodystrophy. Diabetes Care 26: 1819-1824. [Crossref]

3. Guillin-Amarella C, Sanchez-Iglesias S, Castro-Pais A (2016) Type 1 familial partial lipodystrophy: understanding the Köbberling syndrome. Endocrine 54: 411-421.

4. Iwanishi M, Kusakabe T, Azuma C (2019) Clinical characteristics of 15 female Japanese patients with partial lipodystrophy identified in a diabetic outpatient clinic. Diabetes Updates 5.

5. Iwanishi M, Ito-Kobayashi J, Washiyama M (2018) Clinical Characteristics, Phenotype of Lipodystropy and genetic analysis of six japanese female patients with partial lipodystrophy in a diabetic outpatient clinic. Intern Med 57: 2301-2313.

6. DeFronzo RA (2009) From the Triumvirate to the ominous octet: A new paradigm for the treatment of type 2 diabetes mellitus. Diabetes 58: 773-795

7. Abdul-Ghani M, DeFronzo R (2017) Is it time to change the type 2 diabetes treatment paradigm? Yes! GLP-1 Ras should replace metformin in the type 2 diabetes algorithm. Diabetes Care 40: 1121-1132.

8. Iwanishi M, Azuma C, Tezuka Y (2018) Possibility of switching from liraglutide to oral hypoglycemic agents as a useful therapeutic option for obese Japanese type 2 diabetics. Diabetes Management 8: 109-116.

9. Oliveria J, Lau E, Carvalho D (2017) Glucagon-like peptide-1 analogues-an efficient therapeutic option for the severe insulin resistance of lipodystrophic syndromes: two case reports. Journal of Medical Case Reports 11: 12.

10. Komada H, Sakaguchi K, Takeda K (2011) Age-dependent decline in $\beta$-cell function assessed by an oral glucose tolerance test-based disposition index. J Diabetes Investig 2: 293-296. [Crossref]

11. Matsuda M, DeFronzo RA (1999) Insulin sensitivity indices obtained from oral glucose tolerance testing: comparison with the euglycemic insulin clamp. Diabetes care 22: 1462-1470.

12. Matsuda M (2010) Measuring and estimating insulin resistance in clinical and research settings. Nutrition, Metabolism \& Cardiovascular Diseases 20: 79-86.
13. Yamada C, Mitsuhashi T, Hiratsuka N (2011) Optimal reference interval for homeostasis model assessment of insulin resistance in a Japanese population. J Diabetes Investig 2: 373-376.

14. Takahara M, Katakami N, Kaneto H (2013) Distribution of the matsuda index in Japanese healthy subjects. J Diabetes Investig 4: 369-371.

15. Araujiro-Vilar D, Santini F (2018) Diagnosis and treatment of lipodystrophy: a step-bystep approach. Journal of Endocrinological Investigation 42: 61-73.

16. Iwanishi M, Ebihara K, Kusakabe T (2009) Clinical characteristics and efficacy of pioglitazone in a Japanese diabetic patient with an unusual type of familial partial lipodystrophy. Metab Clin Exp 58: 1681-1687.

17. Brown RJ, Araujo-Vilar D, Cheung PT (2016) The diagnosis and management of lipodystrophy syndrome: a multi-society practice guideline. J Clin Endocrinol Metab 85: 1-14.

18. Jendle J, Nauck MA, Matthews DR (2009) Weight loss with liraglutide, a once-daily human glucagon-like peptide-1 analogue for type 2 diabetes treatment as monotherapy or added to metformin, is primarily as a result of a reduction in fat tissue. Diabetes Obes Metab 11: 1163-1172.

19. Li C-J, Yu Q, Yu P (2014) Changes in liraglutide-induced body composition are related to modifications in plasma cardiac natriuretic peptides levels in obese type 2 diabetic patients. Cardiovasc Diabetol Feb 5: 13-36.

20. Lin Y, Berg AH, Iyengar P (2005) The Hyperglycemia-induced inflammatory response in adipocytes. J Biol Chem 280: 4617-4626.

21. Miyachi Y, Tsuchiya K, Shiba K (2018) A reduced M1-like/M2-like ratio of macrophages in healthy adipose tissue expansion during SGLT2 inhibition. Scientific Reports 8: 16113.

22. Sun K, Kusminski CM, Scherer PE (2011) Adipose tissue remodeling and obesity. $J$ Clin Invest 121: 2094-2101. [Crossref]

23. Kahn CR, Wang G, Lee KY (2019) Altered adipose tissue and adipocyte function in the pathogenesis of metabolic syndrome. J Clin Invest 129: 3990-4000.

24. Shulman GI (2014) Ectopic Fat in insulin resistance, Dyslipidemia, and Cardiometabolic disease. N Engl J Med 371: 1131-41.

25. Pinnick KE, Nicholson G, Manolopoulos KN (2014) Distinct developmental profile of lower-body adipose tissue defines resistance against obesity-Associated metabolic complication. Diabetes 63: 3785-3797.

26. Melvin A, Adams C, Flanagan C (2017) Roux-en-Y Gastric Bypass Surgery in the Management of Familial Partial Lipodystrophy Type 1. J Clin Endocrinol Metab 102: 3616-3620.

27. Li Y, Du J, Zhu E (2018) Liraglutide suppresses proliferation and induces adipogenic differentiation of 3T3-L1 cells via the Hippo-YAP signaling pathway. Mol Med Rep 17: 4499-4507.

28. Jiang Y, Wang Z, Ma B (2018) GLP-1 Improves Adipocyte insulin sensitivity following induction of endoplasmic reticulum stress. Front Pharmacol 9: 1168.

29. Lee YS, Park MS, Choung JS (2012) Glucagon-like peptide-1 inhibits adipose tissue macrophage infiltration and inflammation in an obese mouse of diabetes. Diabetologic 55: 2456-2468.

30. Takabe M, Matsuda T, Horota Y (2012) C-peptide response to glucagon challenge is correlated with improvement of early insulin secretion by liraglutide treatment Diabetes Res Clin Pract 98: e32-e35.

31. Banning F, Rottenkolber M, Freibothe I (2017) Insulin secretory defect in familial partial lipodystrophy type 2 and successful long term treatment with a glucagon-like peptide 1 receptor agonist. Diabet Med 34: 1792-1794.

32. Vilsboll T, Bain SC, Leiter LA (2018) Semaglutide, reduction in glycated haemoglobin and the risk of diabetic retinopathy. Diabetes Obes Metab 20: 889-897.

33. Jampol, LM, Glassman AR, Sun J (2020) Evaluation and care of patients with diabetic retinopathy. $N$ Engl J Med 382: 1629-1637.

34. UK Prospective Diabetes Study (UKPDS) Group (1998) Intensive blood-glucose control with sulphonylureas or insulin compared with conventional treatment and risk of complications in patients with type 2 diabetes (UKPDS 33). Lancet 352: 837-853.

35. Gaede P, Lund-Andersen H, Parving HH (2008) Effect of a multifactorial intervention on mortality in Type 2 diabetes. N Engl J Med 358: 580-591.

36. Ueki K, Sasako T, Okazaki Y (2017) Effect of an intensified multifactorial intervention on cardiovascular outcomes and mortality in type 2 diabetes (J-DOIT3): an open-label, randomized controlled trial. Lancet Diabetes Endocrinol 5: 951-964. 
Iwanishi M (2021) Beneficial effects of long-term liraglutide monotherapy on glycemic control and diabetic microangiopathies in a female Japanese diabetic patient with familial partial lipodystrophy 1

37. Holman RR, Paul SK, Bethel MA (2008) 10-Year Follow-up of intensive glucose control in type 2 Diabetes. N Engl J Med 359: 1577-1589.

38. Silva PS, Cavallerano JD, Sun JK (2010) Effect of systemic medications on onset and progression of diabetic retinopathy. Nat Rev Endocrinol 6: 494-507.

39. Fujita H (2014) The protective roles of GLP-1R signaling diabetic nephropathy: possible mechanism and therapeutic potential. Kidney Int 85: 579-589.

40. Marso SP, Daniels GH, Brown-Frandsen K (2016) Liraglutide and cardiovascular outcomes in type 2 diabetes. $N$ Engl J Med 375: 311-22. [Crossref]

41. Mann JF, Orsted DD, Brown-Frandsen K (2017) Liraglutide and renal outcomes in type 2 diabetes. $N$ Engl J Med 377: 839-848.
42. Rakipovski G, Rolin B, Nohr J (2018) The GLP-1 analogs liraglutide and semaglutide reduce atherosclerosis in ApoE-/- and LDLr-/- Mice by a mechanism that includes inflammatory pathways. J Am Coll Cardiol Basic Trans Science 3: 844-857.

43. Koshibu M, Mori Y, Saito T (2019) Anti-atherogenic effects of liraglutide in hyperglycemic apolipoprotein E-null mice via AMP-activated protein kinase independent mechanisms. Am J Physiol Endocrinol Metab 316: E895-E907.

44. Xing Y, Chen J, Zhao L (2020) Analysis of the effect of liraglutide on glycemic variability in patients with type 2 diabetes. Endocr J Jan 28.

45. Ceriello A, Monnier L, Owens D (2019) Glycaemic variability in diabetes: clinical and therapeutic implications. Lancet Diabetes Endocrinol 7: 221-230.

Copyright: (2021 Iwanishi M. This is an open-access article distributed under the terms of the Creative Commons Attribution License, which permits unrestricted use, distribution, and reproduction in any medium, provided the original author and source are credited. 